$01,12,13$

\title{
Особенности электрической проводимости наноостровковых металлических пленок
}

\author{
(C) С.В. Томилин ${ }^{1}$, В.Н. Бержанский ${ }^{1}$, Е.Т. Милюкова ${ }^{1}$, O.А. Томилина ${ }^{1}$, А.С. Яновский ${ }^{2}$ \\ ${ }^{2}$ Научно-исследовательский центр функциональных материалов и нанотехнологий, \\ Физико-технический институт, Крымский федеральный университет им. В.И. Вернадского, \\ Симферополь, Россия \\ ${ }^{2}$ Запорожский национальный университет, \\ Запорожье, Украина \\ E-mail: tomilin_znu@mail.ru; \\ (Поступила в Редакцию 17 ноября 2017 г.)
}

\begin{abstract}
Представлены результаты исследования структурных перколяционных переходов в сверхтонких металлических пленках при термоактивированной грануляции. Показано, что с уменьшением эффективной толщины пленки Au/GGG возрастает степень ее гранулированности, при этом с помощью РЭМ-анализа наглядно продемонстрирована структура пленки при перколяционном переходе. Также в работе проведен анализ возможных причин, механизмов, и моделей активационной электрической проводимости в островковых металлических нанопленках. Рассмотрены вопросы, связанные со статистическим распределением межостровковых промежутков по ширине.
\end{abstract}

Работа выполнена при финансовой поддержке Министерства образования и науки Российской Федерации в рамках базовой части государственного задания (Проект № 3.7126.2017/БЧ).

DOI: $10.21883 /$ FTT.2018.07.46106.330

\section{1. Введение}

В многочисленных экспериментальных работах показано, что характер проводимости наноразмерных материалов существенно отличается от проводимости гомогенных макрообразцов из того же материала. Установлено, например, что температурная зависимость проводимости наноостровковых металлических пленок существенно отличается от аналогичной зависимости объемных образцов и носит активационный характер [1-4]. Данная статья посвящена рассмотрению вопросов, связанных с особенностями активационной проводимости островковых пленок в целом и в частности является результатом теоретического анализа и обобщения экспериментального материала статей $[5,6]$.

В наших предыдущих работах $[5,6]$ приведены результаты экспериментальных исследований изменения проводимости наноостровковых пленок с различной эффективной толщиной $h_{\mathrm{eff}}$ в диапазоне температур от комнатной до $300^{\circ} \mathrm{C}$ для таких металлов, как $\mathrm{Sn}, \mathrm{Al}$, $\mathrm{Cu}, \mathrm{Fe}, \mathrm{Ni}$, Ti и Pt. В них показано что температурная зависимость проводимости сверхтонких и островковых металлических пленок при первичном нагреве в общем случае имеет $N$-образный вид (две области активационного роста проводимости, разделенные областью спада проводимости).

На примере наноостровковой пленки титана (рис. 1) видно, что на начальном этапе нагревания (цикл 1) наблюдается рост проводимости, который носит активационный характер. Далее при определенной температуре наблюдается спад проводимости, вызванный процессами грануляции пленки (образование островковой структуры) вследствие тепловой активации поверхностной диффузии [6]. Когда грануляция пленки завершается полностью, наблюдается повторный активационный рост проводимости. Здесь следует обратить внимание, что после грануляции пленки энергия активации проводимости существенно возросла. Таким образом, энергия активации существенно зависит от степени гранулированности, то есть от расстояния между островками.

При повторном нагревании образца пленки Ті (цикл 2) область спада проводимости отсутствует (пленка полностью гранулировалась при первичном нагреве), однако общий характер активационной проводимости сохраняется.

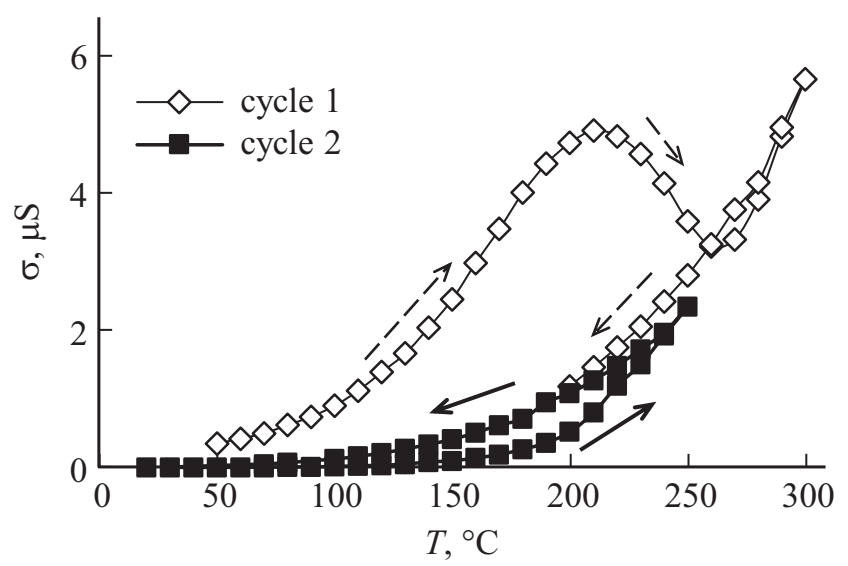

Рис. 1. Температурная зависимость проводимости островковой пленки Тi [6]. 
Существует множество работ, посвященных исследованию проводимости сверхтонких и наноостровковых (гранулированных) пленок. Однако разные авторы, опираясь на общеизвестное понятие активационного процесса, используют совершенно разные модели его математического описания. Так, например, в работе [4] утверждается, что в случае, когда основным механизмом электронного перехода между островками является термоэлектронная эмиссия, температурная зависимость проводимости должна иметь вид (формула Минна)

$$
\sigma=\frac{B e T}{k_{\mathrm{B}}} a \exp \left(-\frac{\varphi-\gamma e^{3} / a}{k_{\mathrm{B}} T}\right),
$$

где $B-$ постоянная, характерная для конкретной пленки, $T$ - абсолютная температура, $e-$ элементарный заряд, $k_{\mathrm{B}}-$ постоянная Больцмана, $a-$ расстояние между островками, $\varphi$ - работа выхода электронов из массивного образца.

Член $\gamma e^{2} / a$ представляет собой вклад сил изображения. Если расстояние а достаточно мало, то этот вклад составляет несколько электронвольт и разность $\varphi-\gamma e^{2} a$ может становиться весьма малой. В этом случае проводимость будет значительно выше.

В случае учета барьерного туннелирования температурная зависимость проводимости также носит активационный характер и имеет вид (соотношение Нейгебауэра и Уэбба) [4]:

$$
\sigma=\frac{A \sqrt{2 m \varphi}}{h^{2} d} a \exp \left(-\frac{4 \pi l}{h} \sqrt{2 m \varphi}\right) B \exp \left(-\frac{e^{2} / \varepsilon r}{k_{\mathrm{B}} T}\right),
$$

где $A$ и $B-$ постоянные; $\varphi-$ потенциальный барьер между островками, приближенно равный работе выхода электронов из массивного образца с учетом сил изображения; $e-$ элементарный заряд; $m-$ эффективная масса электрона; $\varepsilon$ - диэлектрическая проницаемость, величина которой практически находится в пределах между значениями диэлектрических проницаемостей вещества подложки и вакуума; $r-$ средний линейный размер островка.

В работе [7] автор опирается на закон Мотта для прыжковой проводимости:

$$
\sigma=\sigma_{0} \exp \left\lfloor-\left(T_{0} / T\right)^{1 / 4}\right\rfloor,
$$

где $T_{0}=\gamma_{p} \alpha^{3} / N_{\mathrm{F}}, \alpha-$ радиус локализации, $N_{\mathrm{F}}-$ плотность состояний на уровне Ферми, $\gamma_{p}$ - числовой коэффициент.

При этом автор в [7] не уточняет область применимости данного закона с точки зрения типа проводящего материала и его мерности.

В работе [1] утверждается, что зависимость удельной поверхностной проводимости металлических пленок от температуры имеет полупроводниковый характер проводимости. Резкий рост проводимости с увеличением температуры свидетельствует об активационном характере процесса. Соответственно температурную зависимость проводимости островковых и гранулированных пленок авторы объясняют в рамках прыжковой проводимости с переменной длиной прыжка. Величина такой проводимости определяется вероятностью туннелирования носителей между островками и вероятностью активационного теплового перескока:

$$
\sigma \sim \exp \left(-\frac{L}{\lambda}-\frac{E}{k T}\right)
$$

где $L-$ длина прыжка, $\lambda=\hbar /(m W)^{0.5}$ - длина спада волновой функции электрона в диэлектрике, который разделяет металлические островки, $m$ - масса электрона, $W$ - высота туннельного барьера, $E-$ энергия активации.

Как видно, в данном случае рассматривается произведение вероятностей двух событий: туннелирования электронов между островками и активационного перескока, при этом форма барьера рассматривается как прямоугольная. Однако для одного электрона может осуществляться только один процесс. Поэтому необходимо рассматривать сумму вероятностей двух несовместных событий, что исключает вероятность одновременности процессов туннелирования и перескока.

Таким образом, вопрос об однозначности модели и механизма переноса заряда в наноостровковых пленках остается актуальным и однозначно не решенным. Авторами данной статьи предлагается модель прыжковой проводимости, для описания механизма перехода электронов в вакуумном промежутке между металлическими островками.

\section{2. Модель прыжковой проводимости металлических островковых пленок}

C точки зрения структуры островковая пленка представляет собой ансамбль дискретных наночастиц размещенных на поверхности подложки [8].

При исследовании проводимости островковых пленок подложка, как правило, выполнена из диэлектрического материала, а промежутки между металлическими островками заполнены сильно разреженной атмосферой или инертным газом (технический вакуум). С этой точки зрения можно рассматривать островковую пленку как набор последовательных проводников, разделенных вакуумными промежутками. На рис. 2 представлены РЭМ-снимки морфологии поверхности пленки золота на подложке из гадолиний-галлиевого граната (Au/GGG).

Синтез металлического покрытия Au/GGG осуществлялся методом термического вакуумного напыления при давлении остаточных газов не более $5 \cdot 10^{-4} \mathrm{~Pa}$. При напылении пленки была использована оригинальная методика формирования покрытия с градиентом эффективной толщины $h_{\text {eff. }}$ В данном случае градиент эффективной толщины составлял от $5 \mathrm{~nm}$ („толстый“ участок) и вплоть до нулевой толщины (чистая подложка). 
$a$

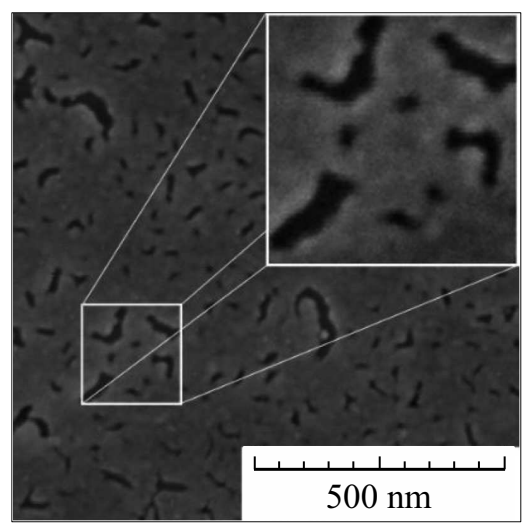

$d$

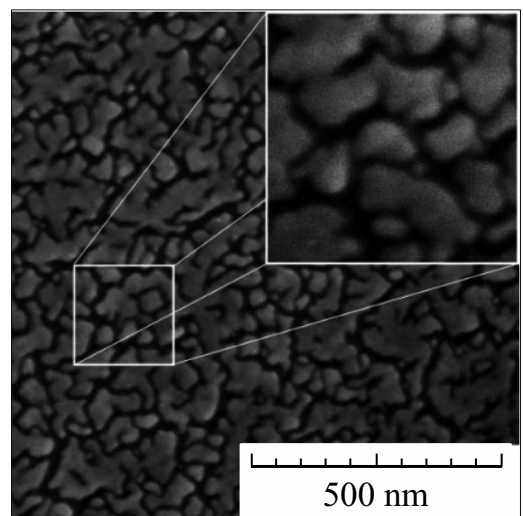

$b$

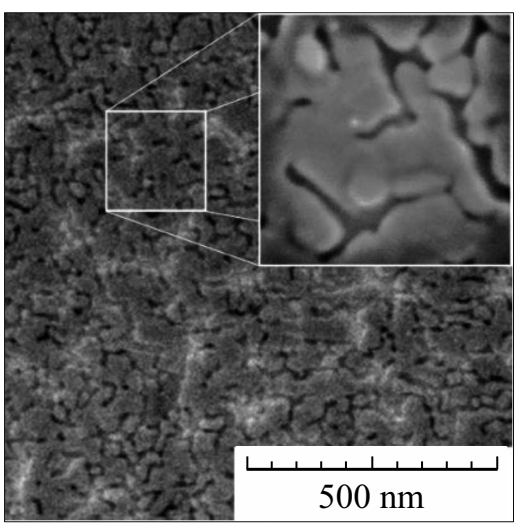

$e$

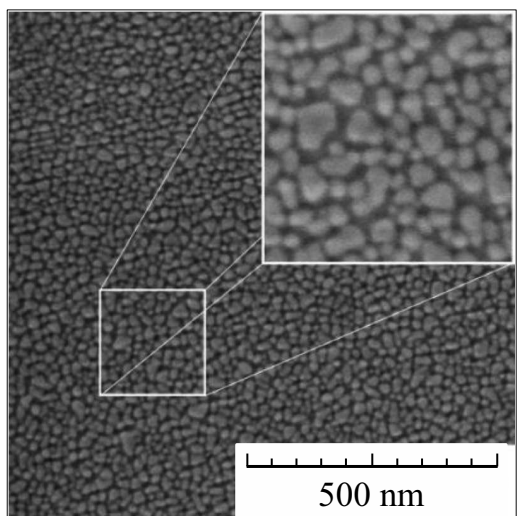

C

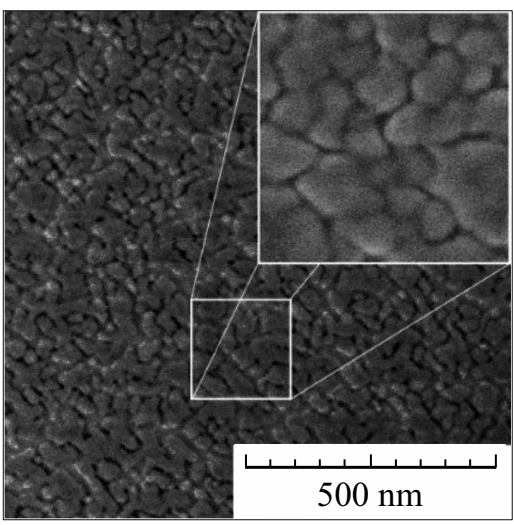

$f$

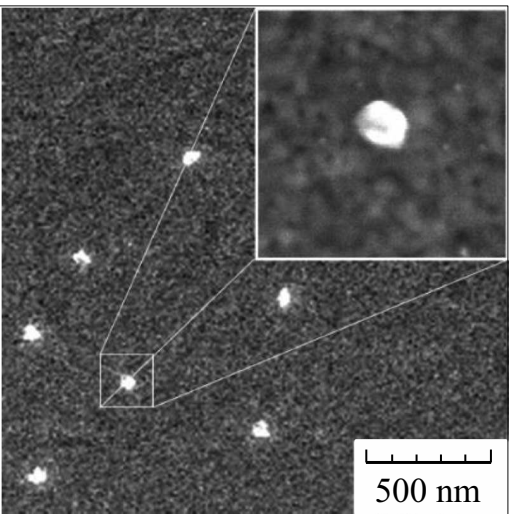

Рис. 2. Пленка $\mathrm{Au} / \mathrm{GGG}$ с градиентом $h_{\mathrm{eff}}$ после отжига при $450^{\circ} \mathrm{C}$ в течение $120 \mathrm{~min} .(a-f)-$ различные участки пленки вдоль градиента. На вставках показаны увеличенные участки $200 \times 200 \mathrm{~nm}$.

После напыления пленка подвергалась термоактивированной грануляции (ТАГ) методом отжига в вакууме при температуре $450^{\circ} \mathrm{C}$ в течение $120 \mathrm{~min}$. Отжиг пленки осуществлялся сразу после напыления без извлечения образца из вакуума. Такая методика позволяет избежать дополнительных загрязнений покрытия. Следует отметить, что температура грануляции в данном эксперименте была значительно ниже температуры плавления золота.

Как видно из представленных снимков, для наиболее „толстого“ участка пленки $\left(h_{\text {eff }} \approx 5 \mathrm{~nm}\right)$ после ТАГ наблюдается образование разрозненных дефектов сплошности покрытия в виде „трещин“ шириной до $20 \mathrm{~nm}$ и длиной до $150 \mathrm{~nm}$ (рис. $2, a$ ). Однако на данном участке покрытие сохраняет свою интегральную сплошность.

На рис. $2, b$ представлен РЭМ-снимок участка пленки с меньшей эффективной толщиной $\left(h_{\mathrm{eff}} \approx 4 \mathrm{~nm}\right)$, чем на предыдущем участке. Видно, что пленка на данном участке содержит многочисленные протяженные дефекты типа „трещина“, которые образуют на поверхности хаотичную сетку. На данном участке дефекты имеют большую протяженность до $200-250 \mathrm{~nm}$ и сложную разветвленную структуру, при этом ширина трещин меньше чем на предыдущем участке (порядка $2-10 \mathrm{~nm}$ ).
Однако следует отметить, что участки металлического покрытия все еще образуют сплошные перколяционные каналы.

На рис. 2, $c$ показана морфология поверхности пленки на участке с $h_{\text {eff }} \approx 3 \mathrm{~nm}$. Видно, что на данном участке дефекты типа „трещина“ образуют сплошную замкнутую сетку. При этом перколяционные каналы разрушаются, образуя дискретные плоские острова неправильной формы. Снимки на рис. 2, $b, c$ наглядно демонстрируют явление перколяционного перехода в структуре покрытия, которое также ранее неоднократно наблюдалось авторами данной работы при исследовании особенностей электропроводности сверхтонких наноструктурированных пленок [5].

Рис. $2, d-f$ демонстрируют последовательные этапы развития островковой структуры покрытия по мере уменьшения его эффективной толщины. Так на рис. $2, d$ $\left(h_{\mathrm{eff}} \approx 2 \mathrm{~nm}\right)$ показаны разрозненные дискретные островки неправильной формы, разделенные „широкими“ промежутками до $20 \mathrm{~nm}$. На рис. 2, $e\left(h_{\mathrm{eff}} \approx 1 \mathrm{~nm}\right)$ так же видны отдельные дискретные островки, однако данные островки имеют меньшие размеры (порядка $5-50 \mathrm{~nm}$ ) и более правильную форму (либо близкую к круговой, либо такую, которая может быть аппроксимирована как 


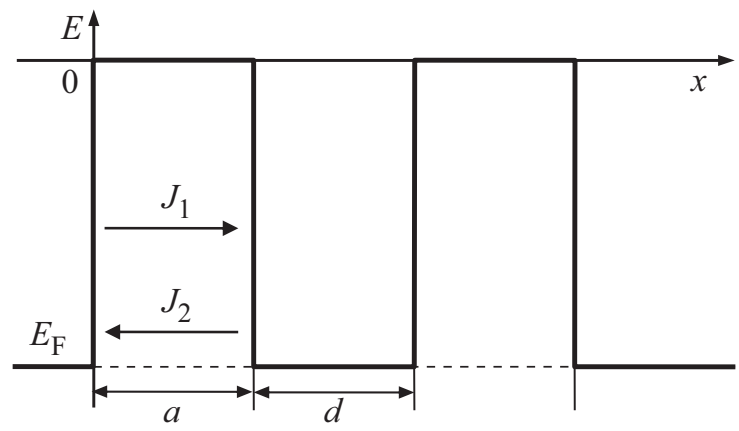

Рис. 3. Одномерная энергетическая диаграмма состояния электрона в наноостровковой пленке (модель прямоугольного барьера).

система двух или более округлых элементов). И наконец на рис. $2, f\left(h_{\mathrm{eff}}\right.$ стремится к $\left.0 \mathrm{~nm}\right)$ мы можем наблюдать отдельные островки с формой близкой к окружности и размером порядка 20-50 nm, отстоящие друг от друга на значительном расстоянии порядка $0.3-1 \mu \mathrm{m}$.

Таким образом видно, что при термогранулляции сверхтонких пленок тип и характер проводимости в первую очередь будет определяться потенциальными барьерами, которые образуются в месте разрыва перколяционных каналов.

Для описания подобных барьеров рассмотрим одномерную энергетическую модель контакта металлвакуум-металл (рис. 3), которая, фактически, отображает энергетическую картину состояния электрона в наноостровковой пленке [9]. Приближенно такое состояние можно описать системой прямоугольных потенциальных ям шириной $d$, разделенных прямоугольными потенциальными барьерами (разрывы между островками). Ширина барьера $a$ соответствует длине прыжка электрона, а его высота равна работе выхода электрона из металлического островка (для больших значений $a$ работа выхода соответствует переходу электрона с уровня ферми $E_{\mathrm{F}}$ на уровень вакуума). Электропроводность в таких системах может одновременно осуществляться по двум механизмам: тепловые перескоки через барьер и квантовое туннелирование сквозь него $[1,10,11]$.

Вероятность теплового перескока через барьер определяется по формуле [12]

$$
f=\exp \left(-\frac{E_{a}}{k_{\mathrm{B}} T}\right),
$$

где $E_{a}-$ энергия активации, $k_{\mathrm{B}} T-$ тепловая энергия.

А вероятность туннелирования электронов сквозь барьер может быть представлена как $[13,14]$

$$
w=\exp \left[-\frac{2}{h} \int_{0}^{a} \sqrt{2 m^{*}[q \varphi(x)-E]} d x\right],
$$

где $m^{*}$ - эффективная масса электрона, $E-$ кинетическая энергия электрона, $\varphi(x)$ - потенциальный барьер, $h$ - постоянная Планка.
Поскольку проводимость может осуществляться по обоим механизмам, то полная вероятность перехода определяется как сумма вероятностей двух несовместимых событий

$$
P(w+f)=w+f-w f .
$$

Соответственно ток, протекающий через барьер

$$
J=J_{0} P .
$$

Коэффициент $J_{0}$ по сути есть ток, который бы протекал через барьер с нулевой шириной и высотой (вероятность перехода равна 1). В общем случае, перенос электрона с одного островка на другой можно представить в виде двух последовательных процессов: эмиссия электрона (тепловая или полевая) с одного металлического островка и захват его другим островком. Из этих соображений выражение для тока $J_{0}$ может быть представлено в виде предэкспоненциального множителя в уравнении Ричардсона-Дешмана $J_{0}=A_{0}(1-\alpha) T^{2}$ [15], где $A_{0}=\frac{4 \pi m^{*} q k_{\mathrm{B}}^{2}}{h^{3}}-$ постоянная Зоммерфельда, $a-$ усредненный по энергиям коэффициент отражения электронов от барьера (для металлов $a \approx 0.5), T-$ абсолютная температура.

Очевидно, что при условии равновесия токи $J_{1}$ и $J_{2}$ (рис. 2), протекающие через барьер будут равны. Для возникновения направленного дрейфового тока образец наноостровковой пленки необходимо поместить во внешнее электрическое поле $E_{0}=U_{0} / S$, где $U_{0}-$ разность потенциалов внешнего поля на концах образца, $S$ - ширина образца.

Внешнее поле приводит к перераспределению заряда внутри островка, что вызывает появление „встроенного “ поля $E_{b i}$. При установлении равновесия напряженность встроенного поля по модулю равна напряженности внешнего $\left(E_{0}=E_{b i}\right)$, поэтому результирующее поле внутри островка равно нулю (Дебаевское экранирование). При этом нескомпенсированные заряды распределяются на противоположных поверхностях островков. Такое перераспределение зарядов приводит к появлению электрического поля внутри барьера $E_{\mathrm{bar}}$ (рис. 4).

Очевидно, что перераспределение зарядов должно приводить к сильной поляризуемости островкового покрытия. То есть островковые пленки должны обладать высокой диэлектрической проницаемостью, что в свою очередь также отмечается рядом авторов [16].

В рамках рассмотренной модели несложно показать, что

$$
E_{\mathrm{bar}}=\frac{\Delta \varphi_{\mathrm{bar}}}{a}=\frac{E_{0}}{2} \frac{d_{1}+d_{2}}{a} .
$$

Тогда полное поле внутри барьера равно сумме внешнего и барьерного полей

$$
E=E_{0}+E_{\mathrm{bar}}=E_{0}\left(\frac{d_{1}+d_{2}}{2 a}+1\right) .
$$




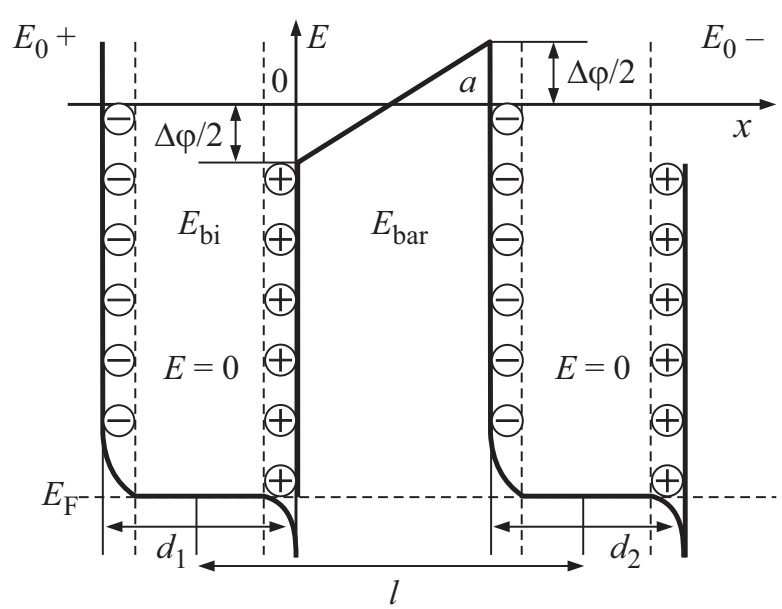

Рис. 4. Распределение потенциала при приложенном внешнем поле.

А разность потенциалов полного поля на барьере определяется как произведение поля на ширину барьера.

$$
\Delta \varphi=E \cdot a=E_{0}\left(\frac{d_{1}+d_{2}}{2}+a\right)=E_{0} l=\frac{U_{0} l}{S} .
$$

Как видно из диаграммы на рис. 4 наличие внешнего поля приводит к смещению энергетических уровней и искажению формы барьера. Такое смещение приводит к возникновению конечной ненулевой разности вероятностей перехода электрона через барьер „слева-направо“ и „справа-налево“ и вызывает возникновение направленного тока. Однако описанная модель не поясняет два экспериментальных факта: во-первых, энергия активации прыжковой проводимости (высота потенциального барьера) существенно меньше работы выхода электрона из металла на уровень вакуума, во-вторых, энергия активации зависит от длины прыжка (ширины барьера).

Рассмотрим действительную форму потенциального барьера. При эмиссии электрона из островка, он собственным электрическим полем индуцирует нескомпенсированный положительный заряд в островке. В результате между островком и электроном возникает кулоновская сила, которую иногда называют силой „зеркального изображения“ $[17,18]$.

При этом потенциал кулоновского электростатического взаимодействия может быть описан гиперболическим уравнением

$$
\varphi_{1}(x)=-k \frac{q}{2\left(x+x_{0}\right)},
$$

где $k=\frac{1}{4 \pi \varepsilon_{0} \varepsilon}-$ коэффициент пропорциональности, $q-$ индуцированный заряд (численно равен модулю заряда электрона), $x$ - расстояние от электрона до поверхности проводника.

Так как потенциал в нуле стремится к минус бесконечности (что не имеет физического смысла), была введена поправка на радиус взаимодействия атомов $x_{0}$. То есть величина $x_{0}$ определяет минимально возможное расстояние, на которое можно приблизить два металла и равняется приблизительно половине межатомного расстояния. Иной физический смысл константы $x_{0}$ заключается в том, что при $x=0$ (поверхность металла) потенциал $\varphi(0)=E_{\mathrm{F}}$ (граничное условие сшивки). Из этого условия несложно показать, что

$$
x_{0}=-\frac{k q}{2 E_{\mathrm{F}}} .
$$

С другой стороны, этот же электрон индуцирует нескомпенсированный заряд в другом островке. Потенциал взаимодействия в этом случае может быть представлен выражением

$$
\varphi_{2}(x)=-k \frac{q}{-2\left(x-a-x_{0}\right)}=-k \frac{q}{2\left(a+x_{0}-x\right)} .
$$

Тогда результирующий потенциал, который действует на электрон между двумя островками, равен сумме потенциалов (12) и (14).

$$
\begin{aligned}
\varphi(x) & =\varphi_{1}+\varphi_{2}=-\frac{k q}{2}\left(\frac{1}{a+x_{0}-x}+\frac{1}{x+x_{0}}\right) \\
& =-\frac{k q}{2} \frac{2 x_{0}+a}{-x^{2}+a x+\left(x_{0}^{2}+a x_{0}\right)}
\end{aligned}
$$

На рис. 5 изображена энергетическая диаграмма, которая иллюстрирует реальную форму потенциального барьера, образованного „зеркальными“ силами электростатического взаимодействия электрона с индуцированными зарядами в островках.

Высота такого барьера $\varphi_{\max }$ и определяет энергию активации проводимости. При условии равновесия

$$
\varphi_{\max }=\varphi(a / 2)=-2 k q \frac{2 x_{0}+a}{a^{2}+4\left(x_{0}^{2}+a x_{0}\right)} .
$$

Соответственно энергия активации

$$
E_{a}=q \varphi_{\max }-E_{\mathrm{F}}=-2 k q^{2} \frac{2 x_{0}+a}{a^{2}+4\left(x_{0}^{2}+a x_{0}\right)}-E_{\mathrm{F}} .
$$

Из анализа диаграммы на рис. 5 можно сделать следующие выводы. Во-первых, высота такого барьера

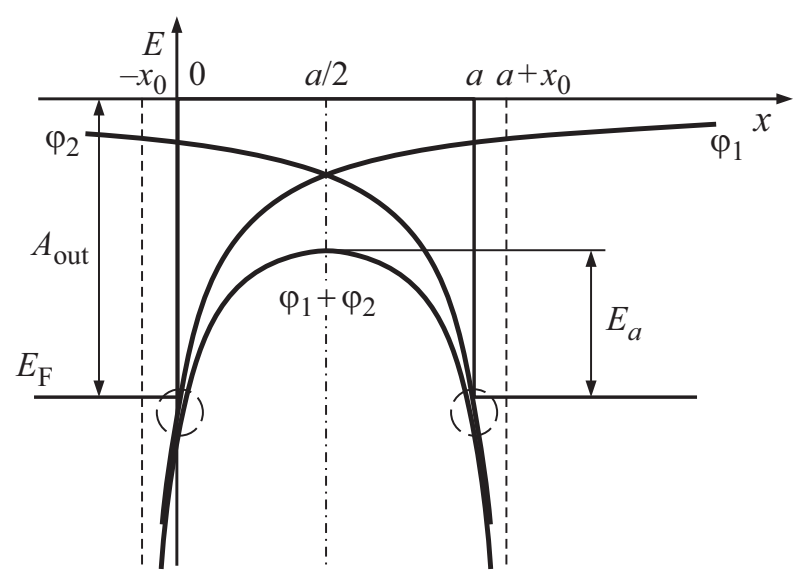

Рис. 5. Форма потенциального барьера, образованного кулоновскими силами „зеркального изображения“. 
гораздо ниже, чем работа выхода электрона из металла на уровень вакуума. Во-вторых, изменение расстояния между островками приводит к изменению не только ширины, но и высоты потенциального барьера (17), что наблюдалось нами экспериментально [5,6]. В-третьих, в результате снижения потенциального барьера в приграничной с островками области образуются потенциальные „карманы“ (на рис. 5 обведены пунктирными окружностями). Такие карманы являются потенциальными ловушками для электронов. Таким образом, в приграничной области островка возникнет избыточная концентрация электронов, поле которых приведет к еще большему снижению высоты потенциального барьера. Глубина такого кармана $q \varphi_{1}(a)$ или $q \varphi_{2}(0)$.

На рис. 6 представлены зависимости высоты потенциального барьера (энергии активации), и вероятность преодоления электроном данного барьера $P(a)$ от ширины барьера (длины прыжка), рассчитанные на основании уравнений (17) и (7) соответственно. В расчетах принято значение уровня Ферми $E_{\mathrm{F}}=-4.23 \mathrm{eV}$ (для $\mathrm{Al}$ ). Как видно из рисунка при значении межостровкового расстояния $a \leq 3.5 \AA$ высота потенциального барьера $E_{a}$ равна нулю, а вероятность перехода электрона соответственно равна 1. При $a>3.5 \AA$ энергия $E_{a}$ резко возрастает и постепенно стремится к постоянному значению, равному работе выхода электрона на уровень вакуума. При этом вероятность перехода электрона через барьер, а соответственно и проводимость, начинают спадать, асимптотически приближаясь к нулю. Данный эффект и наблюдается экспериментально при грануляции пленок и увеличении промежутков между островками.

Как уже отмечалось, для возникновения направленного дрейфа электронов необходимо наличие внешнего электрического поля. При этом распределение потенциала данного поля внутри барьера с учетом (11) может быть описано уравнением

$$
\varphi_{U_{0}}=\frac{U_{0}}{S} x-\frac{U_{0} l}{2 S} .
$$

А полный потенциал определяется, как сумма результирующего потенциала сил зеркального изображения (15) и потенциала внешнего поля (18)

$$
\varphi_{\text {tot }}=-\frac{k q}{2} \frac{2 x_{0}+a}{-x^{2}+a x+\left(x_{0}^{2}+a x_{0}\right)}+\frac{U_{0}}{S} x-\frac{U_{0} l}{2 S} .
$$

На рис. 7 представлены результаты расчета потенциалов зеркального изображения и результирующего потенциального барьера при наложении внешнего поля. Расчеты проводились для вакуумного барьера шириной $a=2 \mathrm{~nm}$ при приложенной внешней разности потенциалов $U_{0}=2 \mathrm{~V}$. Положение уровня Ферми $E_{\mathrm{F}}=-4.23 \mathrm{eV}$.

Таким образом, показано, что высота потенциального барьера (энергия активации прыжковой проводимости) зависит от расстояния между островками. Однако в силу вероятностных процессов образования островковых зародышей расстояние между ними является величиной

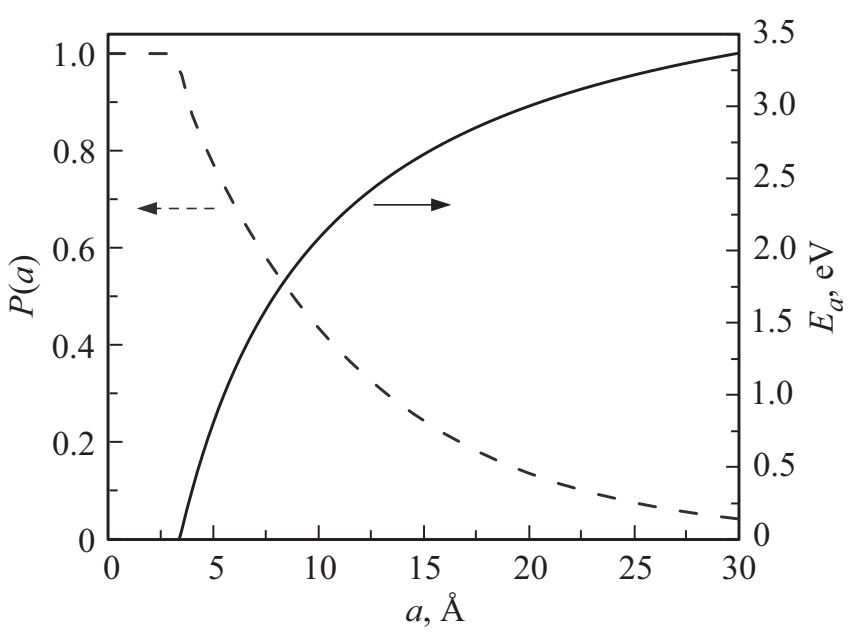

Рис. 6. Зависимость высоты потенциального барьера от его ширины и соответствующая вероятность преодоления электроном данного барьера.

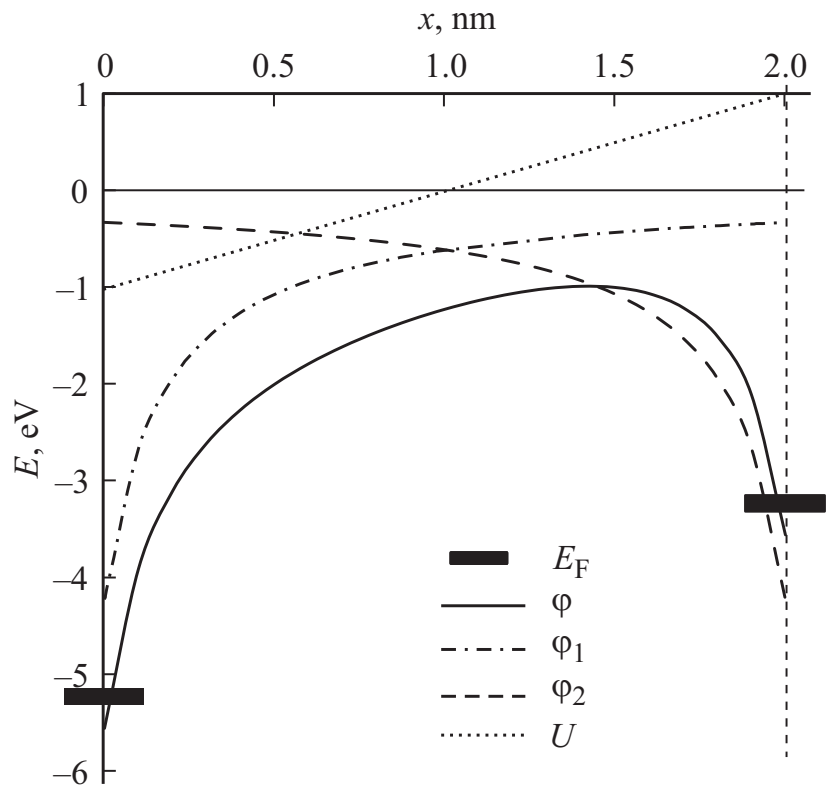

Рис. 7. Распределение потенциала на барьере при наложении внешнего поля: $E_{\mathrm{F}}-$ положение уровня Ферми, $\varphi_{1}-$ потенциал взаимодействия электрона с „левым“ островком, $\varphi_{2}$ потенциал взаимодействия электрона с „правым“ островком, $U-$ потенциал внешнего электрического поля, $\varphi-$ результирующий потенциальный барьер.

не постоянной, а имеет статистический разброс. В этом случае среднее значение энергии активации можно определить как

$$
\left\langle E_{a}\right\rangle=\frac{\int_{0}^{a_{\max }}\left[q \varphi_{\max }(a)-E_{\mathrm{F}}\right] n(a) d a}{\int_{0}^{a_{\max }} n(a) d a},
$$

где $\varphi_{\max }(a)$ - функция зависимости точки максимума потенциального барьера от его ширины; 
$n(a) \approx N(a)^{1 / 2}-$ функция распределения числа барьеров по $a$ в направлении тока $J ; N(a)-$ функция распределения числа барьеров по всей площади образца.

Вид функции распределения барьеров $N(a)$ получим из следующих соображений. Распределение островков по размерам $N(r)$ может быть получено из анализа микроснимков (электронная, зондовая микроскопия и т.д.) посредством аппроксимации экспериментальных статистических данных. Как показывают практические результаты вид распределения может иметь как симметричную форму (нормальное распределение Гаусса), так и иметь асимметрию в сторону больших либо меньших значений аргумента (распределения Релея, Лифшица-Слезова и т.д.) [19-22]. В общем случае конкретный вид функции распределения может быть описан полиномом степени $n$.

Рассмотрим случай, когда распределение островков по размерам может быть описано функцией ЛифшицаСлезова [20]

$$
N(r)=\frac{2 N_{0}}{r_{a v}} P(u),
$$

где $N_{0}$ - концентрация островков $u=r / r_{a v}$

$$
P(u)=\left\{\begin{array}{l}
\frac{3^{4} e}{2^{5 / 3}} \frac{u^{2} \exp [-1 /(1-u(2 / 3))]}{(u+3)^{7 / 3}(3 / 2-u)^{11 / 3}}, \quad u<3 / 2 \\
0, \quad u \geq 3 / 2
\end{array} .\right.
$$

Таким образом, распределение островков по размерам представляется как функция радиусов $N(r)=f(r)$. Учитывая, что рост островков осуществляется за счет диффундирующих атомов и приняв во внимание, что вероятность диффузии во всех направлениях одинакова, несложно найти соотношение между радиусом островка и его зоной захвата

$$
r=\left(\frac{3}{4} h\right)^{1 / 3} R^{2 / 3},
$$

где $h$ - эффективная толщина пленки, $R$ - радиус зоны захвата (примерно равен диффузионной длине пробега атома).

На практике, как правило, наблюдается не максимальное, а частичное заполнение поверхности островками. В таком случае на расстояния между островками $l$ будет влиять фактор заполнения $k=N_{0} / N_{\max }(k \geq 1)$, и соответственно $l=R / k$. Подставив полученное выражение (22) в (21) получим функцию распределения расстояний между островками в виде

$$
N(l)=f\left(\left[\frac{3}{4} h\right]^{1 / 3} k^{2 / 3} l^{2 / 3}\right)=f\left(A l^{2 / 3}\right),
$$

где

$$
A=\left(\frac{3}{4} h\right)^{1 / 3}\left(\frac{N_{0}}{N_{\max }}\right)^{2 / 3}
$$

Промежуток между островками (длину прыжка) можно представить как

$$
a=l-2 r=l-2 A l^{2 / 3} .
$$

Введя в (24) замену $l=y^{3}$ получим кубическое уравнение

$$
y^{3}-2 A y^{2}-a=0 .
$$

Анализ данного уравнения показывает, что оно имеет один действительный положительный корень. Решая его методом Кардана получим

$$
\begin{gathered}
y=v_{1}+v_{2}+\frac{2}{3} A, \\
l=\left(v_{1}+v_{2}+\frac{2}{3} A\right)^{3} .
\end{gathered}
$$

Здесь члены $v_{1}$ и $v_{2}$

$$
\begin{aligned}
& v_{1}=\left[\left(\frac{2 A}{3}\right)^{3}+\frac{a}{2}+\left[a\left(\frac{2 A}{3}\right)^{3}+\frac{a^{2}}{4}\right]^{1 / 2}\right]^{1 / 3}, \\
& v_{2}=\left[\left(\frac{2 A}{3}\right)^{3}+\frac{a}{2}-\left[a\left(\frac{2 A}{3}\right)^{3}+\frac{a^{2}}{4}\right]^{1 / 2}\right]^{1 / 3} .
\end{aligned}
$$

Таким образом, подставляя (26) в (23) получим окончательное распределение числа потенциальных барьеров по ширине

$$
N(a)=f\left(A\left[v_{1}+v_{2}+\frac{2}{3} A\right]^{2}\right),
$$

где $f$ - есть функция распределения Лифшица-Слезова.

Поскольку данное распределение выведено из общих соображений структурной геометрии пленки, то уравнение (28) может быть распространено на произвольную функцию $f(r)$. Переход от функции распределения островков к функции распределения барьеров в таком случае осуществляется заменой аргумента $r$ на аргумент

$$
A\left[v_{1}+v_{2}+\frac{2}{3} A\right]^{2} .
$$

Вид распределения (28), рассчитанного для пленок с различной эффективной толщиной, для которых распределение островков по размерам описывается функцией Лифшица-Слезова, показан на рис. 8.

Из вида данного распределения можно сделать следующие заключения. Во-первых, максимум распределения с увеличением эффективной толщины пленки смещается в сторону уменьшения $a$. То есть чем толще пленка, тем уже потенциальные барьеры между островками и тем меньше энергия активации прыжковой проводимости, что в свою очередь прекрасно согласуется с результатами эксперимента. Во-вторых, с увеличением параметра $a$ 


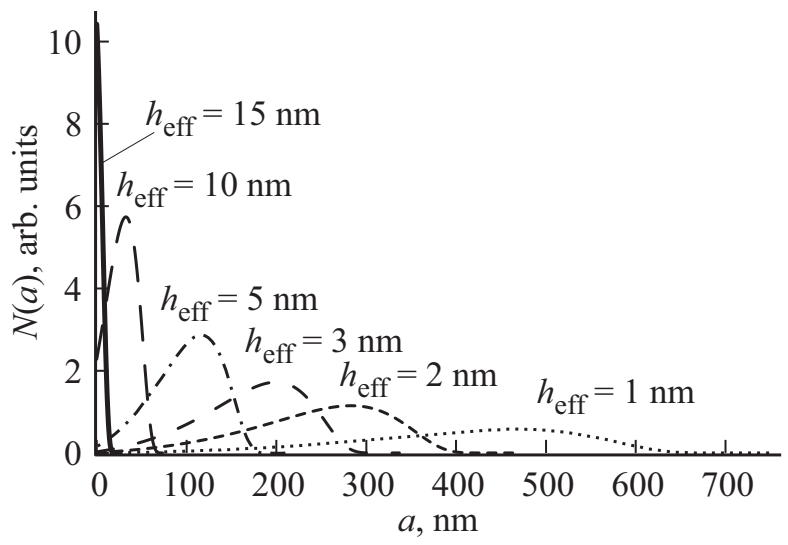

Рис. 8. Распределение числа потенциальных барьеров по ширине $N(a)$ в пленках с разной эффективной толщиной (для случая функции Лифшица-Слезова).

кривая не плавно (асимптотически), а достаточно резко спадает вплоть до нулевого значения, причем, чем толще пленка, тем круче спад и тем меньше возможная ширина барьеров. И, в-третьих, следует обратить внимание, что в распределении имеет место конечное число „нулевых“ барьеров (шириной $0 \mathrm{~nm}$ ), и чем толще пленка, тем их больше. Для пленок толщиной $h=15 \mathrm{~nm}$ и более, „нулевые“ барьеры составляют максимум распределения. То есть для пленок с относительно большой эффективной толщиной имеет место явление сращивания островков, что в свою очередь так же подтверждается на практике и, вообще говоря, является основой механизма островкового роста пленок. Анализ показал, что для пленок с толщиной $h=19 \mathrm{~nm}$ и более (в случае если распределение островков по размерам для аналогичных пленок с меньшей $h_{\text {eff }}$ описывается функцией Лифшица-Слезова), „ненулевые“ барьеры отсутствуют, то есть пленки являются сплошными и не образуют островковую структуру, что так же подтверждено на практике [23,24].

\section{3. Выводы}

При рассмотрении механизма проводимости металлических островковых пленок в виде перехода (прыжка) электрона с одного островка на соседний минуя вакуумный промежуток (потенциальный барьер) необходимо учитывать два механизма: тепловой перескок через потенциальный барьер и квантовое туннелирование сквозь него. При этом общая вероятность такого перехода должна определяться как сумма вероятностей двух несовместных событий.

Рассмотрение природы потенциального барьера между наноостровками с точки зрения электростатических сил зеркального изображения позволяет качественно и количественно показать взаимосвязь между шириной потенциального барьера и его высотой (энергией активации прыжковой проводимости). При этом показано, что при относительно близком расположении наноостровков высота потенциального барьера, образованного вакуумным промежутком, существенно ниже чем работа выхода электрона из металла на энергетический уровень вакуума.

Статистический разброс межостровковых расстояний (ширины потенциального барьера) приводит к необходимости рассмотрения вопроса о функции распределения данных расстояний по величине. Отыскание вида данной функции из общих соображений структурной геометрии пленки на основании диффузионной теории формирования наноостровков приводит к следующим заключениям. Во-первых, максимум распределения с увеличением эффективной толщины пленки смещается в сторону уменьшения $a$. Во-вторых, в распределении имеет место конечное число „нулевых“ барьеров (шириной $0 \mathrm{~nm}$ ), и чем толще пленка, тем их больше, т.е. для пленок с относительно большой эффективной толщиной имеет место явление сращивания островков. Для пленок с толщиной $h_{\mathrm{eff}}=19 \mathrm{~nm}$ и более (в случае если распределение островков по размерам для аналогичных пленок с меньшей $h_{\text {eff }}$ описывается функцией Лифшица-Слезова), „ненулевые“ барьеры отсутствуют, то есть пленки являются сплошными и не образуют островковую структуру.

\section{Список литературы}

[1] А.П. Болтаев, Н.А. Пенин, А.О. Погосов, Ф.А. Пудонин. ЖЭТФ 126, 954 (2004).

[2] A. Ito, H. Masumoto, T. Goto. Mater. Transactions 49, 158 (2008).

[3] J.S. Agustsson, U.B. Arnalds, A.S. Ingason, K.B. Gylfason, K. Johnsen, S. Olafsson, J.T. Gudmundsson. J. Phys.: Conf. Ser. 100, 082006 (2008).

[4] Н.А. Абдуллаев, Н.М. Абдуллаев, Х.В. Алигулиева, А.М. Керимова, К.М. Мустафаева, И.Т. Мамедова, Н.Т. Мамедов, С.А. Немов, П.О. Буланчук. ФТП 47, 586 (2013)

[5] С.В. Томилин, В.Н. Бержанский, Е.Т. Милюкова, О.А. Томилина, А.С. Яновский. ФТТ 59, 639 (2017).

[6] С.В. Томилин, В.Н. Бержанский, А.С. Яновский, О.А. Томилина. Поверхность 8, 96 (2016)

[7] М.П. Фатеев. ФТТ 52, 1053 (2010).

[8] В.Б. Лобода, С.Н. Хурсенко. ЖЭТФ 130, 911 (2006).

[9] П.В. Павлов, А.Ф. Хохлов. Физика твердого тела. Высш. шк. М. (2000). 497 с.

[10] J.L.M. Rupp, L.J. Gauckler. Solid State Ionics 177, 2513 (2006).

[11] R.D. Maldonado, A.I. Oliva. Surf. Rev. Lett. 15, 881 (2008).

[12] Ч. Киттель. Введение в физику твердого тела. Под ред. А.А. Гусева. Гл. ред. физ.-мат. лит., М. (1978). 792 с.

[13] Е.Н. Дулов, Ф.Г. Вагизов, М.М. Бикчантаев, А.В. Пятаев, Р.Р.Гайнов. Регистрация спектров альфа-частиц, полупроводниковые детекторы. Казанский (Приволжский) федеральный университет, Казань. (2013). 27 с.

[14] В.Е. Борисенко, А.И. Воробьева, А.Л. Данилюк, Е.А. Уткина. Наноэлектроника: теория и практика 2-е изд. БИНОМ. Лаборатория знаний, М. (2013). 366 с. 
[15] В.И. Светцов, И.В. Холодков. Физическая электроника и электронные приборы. Ивановский гос. хим.-технол. ун-т, Иваново (2008). 494 с.

[16] А.П. Болтаев, Ф.А. Пудонин. ЖЭТФ 134, 587 (2008).

[17] Г.Н. Фурсей. Сорос. образоват. журн. 6, 96 (2000).

[18] А.И. Войтенко, А.М. Габович. ФТТ 43, 2230 (2001).

[19] А.К. Симонян. Изв. НАН Армении, Физика 48, 330 (2013).

[20] И.М. Лифшиц, В.В. Слезов. ЖЭТФ 35, 479 (1958).

[21] В.Г. Дубровский. ФТП 40, 1153 (2006).

[22] Т.Б. Иванова, В.В. Васькин. Вестн. удмуртского ун-та. Компьютерные науки 2, 110 (2009).

[23] S.V. Tomilin, A.S. Yanovsky. J. Nano- Electron. Phys. 4. 01013 (2012).

[24] S.V. Tomilin, A.S. Yanovsky. J. Nano- Electron. Phys. 5, 03014 (2013).

Редактор Т.Н. Василевская 